\title{
Ciclos de discussão de Segurança Alimentar e Nutricional: promovendo a troca de saberes entre sala de aula e a comunidade
}

\author{
Sílvia Oliveira Lopes ${ }^{1}$, Dayane de Castro Morais ${ }^{2}$, Elizangela da \\ Silva Miguel ${ }^{3}$, Paula Torres Trivellato ${ }^{4}$, Luiza Veloso Dutra ${ }^{5}$, \\ Carina Aparecida Pinto ${ }^{3}$, Silvia Eloiza Priore 6
}

\begin{abstract}
Resumo: O objetivo do trabalho é analisar os resultados e reflexões geradas nos encontros do "Ciclo de Discussão de Segurança Alimentar e Nutricional". Nestes Ciclos são compartilhadas as construções da disciplina "Determinantes da Insegurança Alimentar e Nutricional" difundindo e valorizando trabalhados dos pós-graduandos e docentes em temas relacionados à Segurança Alimentar e Nutricional (SAN). A análise dos temas discutidos nos Ciclos, por meio da análise de conteúdo e de similitude, considerou-se as oito diretrizes da Política Nacional de Segurança Alimentar e Nutricional (PNSAN). Foram realizados oito Ciclos, sendo estes anuais. Todas as discussões basearam-se em diferentes temáticas que permearam o contexto da SAN e contemplaram pelo menos um dos eixos das diretrizes da PNSAN. Conclui-se que ações como estas são importantes para estimular o pensamento crítico para além da sala de aula e propiciar a troca de saberes entre universidade e comunidade, consolidando a tríade ensino, pesquisa e extensão.
\end{abstract}

Palavras-chave: Segurança Alimentar e Nutricional. Relações Comunidade-Instituição. Práticas Interdisciplinares.

Área Temática: Educação. Segurança Alimentar.

\section{Discussion cycles of food and nutrition security: promoting the exchange of knowledge between the classroom and the community}

\begin{abstract}
The objective of the work is to analyze the results and reflections generated in the meetings of the "Cycle of Discussion on Food and Nutritional Security". In these cycles the constructions of the discipline are shared "Determinants of Food and Nutritional Insecurity", disseminating and valuing graduate work and documents on themes related to Food and Nutritional Security. The analysis of the topics discussed in Cycles, through content and similarity analysis, is considered as guidelines of the National Food and Nutrition Security Policy (PNSAN). Eight cycles were carried out, the latter being. All discussions are based on different themes that allow the context of the Food and Nutritional Security and include at least one of the axes of the PNSAN guidelines. Conclude that actions like these are important to stimulate critical thinking beyond the classroom and provide an exchange of knowledge between university and community, consolidating high school, research and extension.
\end{abstract}

Keywords: Food and nutrition security. Community-Institutional Relations. Interdisciplinary Practices.

\footnotetext{
${ }^{1}$ Nutricionista, Doutoranda em Ciência da Nutrição pela Universidade Federal de Viçosa (UFV), Travessa Felício Brandi nº0 Apto 202, Centro, Viçosa-Minas Gerais (CEP 36570-097), +55 (31) 98325-7726, silvia.lopes.nut@ hotmail.com

${ }^{2}$ Nutricionista, Pós-doutoranda em Ciência da Nutrição/UFV

${ }^{3}$ Nutricionista, Doutoranda em Ciência da Nutrição/UFV

${ }^{4}$ Nutricionista, Mestre em Agroecologia/UFV

${ }^{5}$ Nutricionista, Doutora em Ciência da Nutrição/UFV, Professora do Centro Universitário de Viçosa/Univiçosa

${ }^{6}$ Nutricionista, Doutora em Nutrição pela Universidade Federal de São Paulo (USP), Professora do Departamento de Nutrição e Saúde/UFV
} 


\section{Ciclos de discusión sobre seguridad alimentaria y nutricional: promover el intercambio de conocimientos entre el aula y la comunidad}

Resumen: El objetivo del trabajo es analizar los resultados y las reflexiones generadas en las reuniones del "Ciclo de debate sobre seguridad alimentaria y nutricional". En estos ciclos se comparten las construcciones de la disciplina "Determinantes de la inseguridad alimentaria y nutricional", difundiendo y valorando el trabajo de posgrado y documentos sobre temas relacionados con la Seguridad Alimentaria y Nutricional. El análisis de los temas discutidos en Ciclos, a través del análisis de contenido y similitud, se considera como directrices de la Política Nacional de Seguridad Alimentaria y Nutricional (PNSAN). Se llevaron a cabo ocho ciclos, siendo este último. Todas las discusiones se basan en diferentes temas que permiten el contexto de la Seguridad Alimentaria y Nutricional e incluyen al menos uno de los ejes de las pautas de PNSAN. Concluya que acciones como estas son importantes para estimular el pensamiento crítico más allá del aula y proporcionar un intercambio de conocimientos entre la universidad y la comunidad, consolidando la escuela secundaria, la investigación y la extensión.

Palabras clave: Seguridad alimentaria y nutricional. Relaciones Comunidad-Institucionales. Practicas interdisciplinarias.

\section{INTRODUÇÃO}

A conquista de políticas públicas de Segurança Alimentar e Nutricional (SAN), assim como a estruturação do próprio conceito, demandou fatos históricos, discussões e envolvimentos sociais e políticos, dados por lutas e debates que refletiam os anseios da população em diferentes épocas. Assim, a construção da SAN é compreendida como movimento e política. A Soberania Alimentar e o Direito Humano à Alimentação Adequada (DHAA) se somam à trajetória da SAN reforçando a importância da alimentação em quantidade e qualidade suficiente e adequada, saudável a todos, promovendo a autonomia alimentar, preservação do meio ambiente, sustentabilidade da produção e a garantia da disponibilidade de alimentos para as gerações futuras (PRIORE et al., 2014; MORAIS; SPERANDIO, 2016; NASCIMENTO, ANDRADE, 2020).

ASAN é compreendida para além das dimensões de acesso e disponibilidade de alimentos. Tratase, do direito à informação cientificamente comprovada e respaldada sobre alimentação saudável, alimentos seguros e adequados, bem como ao acesso a informação sobre direitos e ações públicas (ABRANDH, 2013).

O conceito de SAN no Brasil vem sendo construído ao longo das últimas décadas, a partir de debates, estudos e ações, acompanhando o contexto histórico e político vivenciado (PRIORE et al., 2014). A terminologia de SAN, adotada no país, foi publicada pela Lei Orgânica de Segurança Alimentar e Nutricional (LOSAN) em 2006 (BRASIL, 2006), com inclusão da dimensão nutricional neste contexto. Com esse marco legal, o conceito institucional ganhou visibilidade em políticas, programas governamentais e em frentes de pesquisa, ensino e extensão.

Essa iniciativa institucional trouxe redirecionamento das ações já existentes e a criação de políticas e planos nacionais com enfoque na promoção da SAN. Dentro do campo da discussão e formação em SAN, a LOSAN enfatiza o estímulo ao desenvolvimento de pesquisas e à capacitação de recursos humanos na área (BRASIL, 2006; BURITY et al, 2010).

APolítica Nacional de Segurança Alimentar e Nutricional (PNSAN) de 2010, traz em suas diretrizes a instituição de processos permanentes de educação alimentar e nutricional, pesquisa e formação nas áreas de SAN e DHAA, que são reforçadas no Plano Nacional de Segurança Alimentar e Nutricional (BRASIL, 2010). 
A evolução da SAN no país deveu-se ao envolvimento de diferentes atores, políticos e civis, em diferentes cenários, envolvidos em ampla discussão e debate sobre os princípios e necessidades de garantia de SAN, Soberania Alimentar e DHAA (ABRANDH, 2013; PRIORE et al., 2014). Assim, a comunicação é um veículo de promoção da SAN, através do conhecimento compartilhado e construído em espaços sociais. Nesse sentido, é pertinente a criação de ambientes para disseminação e discussão de conhecimentos sobre SAN em locais públicos, especialmente os acadêmicos, onde se agrupam pessoas com formação em diferentes áreas de atuação.

\section{Histórico de construção do "Ciclo de Discussão de Segurança Alimentar e Nutricional"}

Em 2012, iniciou-se a realização do “Ciclo de Discussão de Segurança Alimentar e Nutricional”, na Universidade Federal de Viçosa (UFV), Campus Viçosa - Minas Gerais. Esta proposta surgiu a partir das discussões geradas na disciplina de pós-graduação intitulada “Determinantes da Insegurança Alimentar e Nutricional" cujos conteúdos abordam a influência de fatores sociais, políticos, ambientais e culturais na SAN, na Soberania Alimentar e no DHAA. Esta disciplina é oferecida pelo Departamento de Nutrição e Saúde, aos pós-graduandos de qualquer programa da UFV e/ou de outra instituição de ensino, pública ou privada, sendo coordenada pela professora responsável por sua criação, com oferta anual. A participação mais contundente é a dos pós-graduandos do Programa de Pós-graduação em Ciência da Nutrição e em Agroecologia, ambos da UFV.

O conteúdo da disciplina considera a leitura, análise crítica e apresentação de materiais considerados fundamentais para o estudo da SAN, incluindo documentos publicados por órgãos nacionais e internacionais, artigos científicos, livros e/ou capítulos de livros, além de artigos oriundos de dissertações e teses relacionadas a SAN, desenvolvidas pelos pós-graduandos, bem como experiências práticas dos pós-graduandos matriculados. Durante a disciplina os pós-graduandos são instruídos a construírem um artigo científico, original ou de revisão, que contemple aspectos da SAN e que seja relacionado ao objetivo geral de sua dissertação ou tese. Este exercício de relacionar o seu respectivo estudo com propósitos da SAN permite ao pós-graduando aprofundar conceitos e entender que, direta ou indiretamente, todos os assuntos têm vertente na SAN. O conteúdo discutido em sala é renovado e atualizado a cada oferta da disciplina.

A partir do entendimento de que é necessária a disseminação de informações construídas em sala de aula surgiu a proposta de expansão das discussões das temáticas geradas na disciplina para além de um grupo restrito, de pós-graduandos e docente, a fim de ampliar e proporcionar debate crítico com a comunidade. Diante desse entendimento, e considerando a necessidade de fortalecer a tríade: ensino, pesquisa e extensão, inclusive na pósgraduação, deu-se início ao planejamento e execução dos encontros do "Ciclo de Discussão de Segurança Alimentar e Nutricional" da UFV. 
O “Ciclo de Discussão de Segurança Alimentar e Nutricional” tem como objetivo promover um espaço para disseminação de conhecimentos sobre temas relacionados a SAN, difundindo e valorizando os trabalhos e as reflexões entre acadêmicos, pesquisadores e demais atores.

A realização dos Ciclos, ao longo destes anos de existência, envolveu a participação de docentes, técnicos, pós-graduandos e graduandos de diferentes Centros de Ciências e Departamentos da UFV, como os de Nutrição, Agronomia, Biologia, Economia Rural, Tecnologia de Alimentos, Educação, e outras áreas relacionadas, bem como de profissionais de diferentes campos de atuação.

\section{OBJETIVO}

Este artigo tem por objetivo analisar os resultados e as reflexões geradas nos encontros do "Ciclo de Discussão de Segurança Alimentar e Nutricional".

\section{METODOLOGIA}

\section{Descrição da prática}

Adisciplina “Determinantes da Insegurança Alimentar e Nutricional” é oferecida no segundo semestre de cada ano letivo e no semestre seguinte, os pós-graduandos matriculados, contribuem com a execução das atividades do Ciclo. Além disso, os pós-graduandos que já cursaram, que pesquisam a temática, que atuam juntamente com estes em seus grupos de trabalho, são convidados a participarem da execução e realização dos encontros.

Os ciclos são constituídos de quatro encontros de aproximadamente três horas cada, ocorridos ao longo de quatro semanas, sendo realizado nas dependências da UFV, Campus Viçosa. O público é heterogêneo, composto por discentes da graduação e pós-graduação, docentes, técnicos de diferentes Departamentos, além de público externo à Universidade.

Cada encontro é conduzido por pelo menos um pós-graduando, um docente ou técnico e um mediador, convidados pelos organizadores. Os convidados, para proferir a discussão no ciclo, são pesquisadores ou atuantes na área de SAN, Soberania Alimentar e DHAA. São realizadas apresentações breves de temas que se relacionam com a SAN pelos convidados e, posteriormente, o mediador destaca questões que emergem das apresentações para debater com o público, visando estimular o pensamento crítico referente à temática, resgatando falas e experiências consideradas importantes de serem frisadas.

Os temas apresentados nos encontros do "Ciclo de Discussão de Segurança Alimentar e Nutricional" são aqueles discutidos na disciplina. A dinâmica do Ciclo permite o desenvolvimento de debates que contribuem para o processo de troca de saberes entre academia e comunidade (Figura 1). A heterogeneidade dos temas que se correlacionam com a SAN facilita o processo de discussão que é mediado durante as apresentações. 


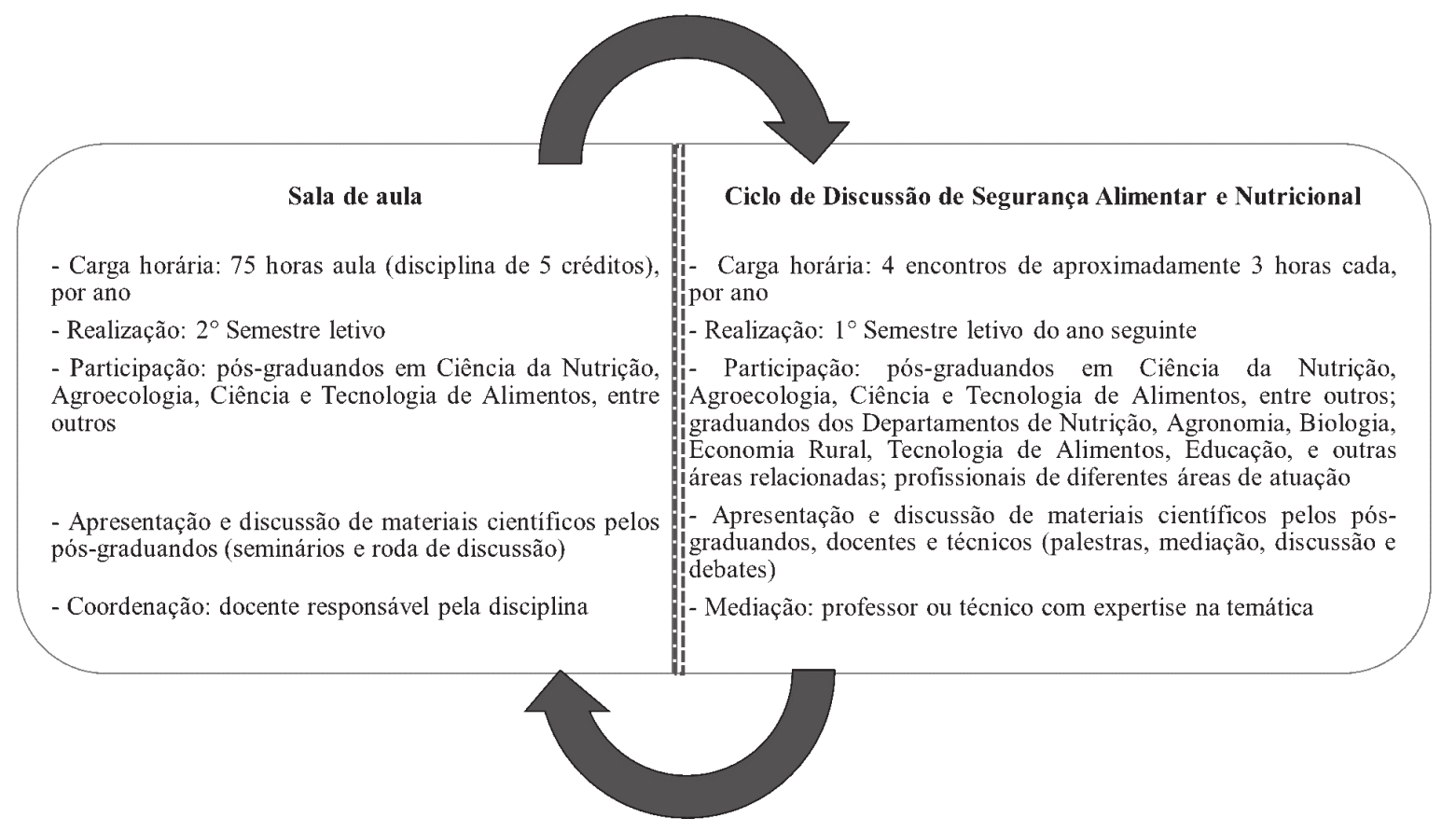

Figura 1. Metodologia proposta para realização do Ciclo de Discussão de Segurança Alimentar e Nutricional considerando a integração entre sala de aula e comunidade (2012-2019).

Fonte: Elaborado pelas autoras.

Ressalta-se que a divulgação dos encontros se dá pelo e-mail institucional da UFV, redes sociais e cartazes fixados em pontos estratégicos da Universidade, no Campus Viçosa e no município. Os encontros do Ciclo são registrados como eventos de extensão, sendo que os participantes (organizadores, palestrantes, mediadores e público inscrito) recebem certificado após a conclusão dos mesmos. Não há financiamento para a execução dos Ciclos, sendo estes realizados com a colaboração dos envolvidos, de forma voluntária.

\section{Análise das discussões dos Ciclos na perspectiva das diretrizes da Política Nacional de Segurança Alimentar e Nutricional}

A fim de auxiliar as análises dos temas abordados, foi realizada uma avaliação conjunta dos eixos que nortearam as discussões dos Ciclos realizados, categorizando os assuntos debatidos a partir das oito diretrizes da PNSAN (BRASIL, 2010).

I - promoção do acesso universal à alimentação adequada e saudável, com prioridade para as famílias e pessoas em situação de Insegurança Alimentar e Nutricional;

II - promoção do abastecimento e estruturação de sistemas sustentáveis e descentralizados, de base agroecológica, de produção, extração, processamento e distribuição de alimentos;

III - instituição de processos permanentes de educação alimentar e nutricional, pesquisa e formação nas áreas de Segurança Alimentar e Nutricional e do Direito Humano à Alimentação Adequada; IV - promoção, universalização e coordenação das ações de Segurança Alimentar e Nutricional voltadas para quilombolas e demais povos e comunidades tradicionais, povos indígenas e assentados da reforma agrária; 
V - fortalecimento das ações de alimentação e nutrição em todos os níveis da atenção à saúde, de modo articulado às demais ações de Segurança Alimentar e Nutricional;

VI - promoção do acesso universal à água de qualidade e em quantidade suficiente, com prioridade para as famílias em situação de insegurança hídrica e para a produção de alimentos da agricultura familiar e da pesca e aquicultura;

VII - apoio a iniciativas de promoção da Soberania Alimentar, Segurança Alimentar e Nutricional e do Direito Humano à Alimentação Adequada em âmbito internacional e a negociações internacionais; VIII - monitoramento da realização do Direito Humano à Alimentação Adequada.

Os assuntos abordados nos ciclos foram agrupados quanto às temáticas comuns. Após esta organização no Microsoft Word ${ }^{\circledR}$ foi realizada a categorização baseada nas diretrizes da PNSAN e, posteriormente, análise de conteúdo segundo proposta de Bardin (2011), a partir de registro escrito por um observador, em cada edição do Ciclo considerando a modalidade temática de avaliação construída por Minayo (2013), que consiste em: préanálise, exploração do material e tratamento dos resultados/inferência/interpretação.

Os dados foram digitados e exportados para o software IRaMuTeQ (Interface do R) desenvolvido por Pierre Ratinaud (2009), para análise multidimensional dos temas e gerenciamento das informações pela análise de similitude. Esta análise baseia-se na teoria dos grafos (MARCHAND, RATINAUD, 2012) que busca identificar as concorrências entre palavras ou expressões indicando conexão entre elas.

\section{RESULTADOS E DISCUSSÃO}

No período de 2012 a 2019, foram realizadas oito edições do "Ciclo de Discussão de Segurança Alimentar e Nutricional”, totalizando 32 encontros. A média de público por encontro foi de 40 pessoas, com destaque para a participação dos cursos de graduação em Nutrição, Economia Doméstica, Agronomia, Economia, Direito, Ciências Sociais, Ciências Biológicas, Licenciatura e Educação do Campo (Licena), Gestão de Cooperativas e Geografia; Cursos de pós-graduação, sobretudo os de Ciência da Nutrição, Agroecologia, Fitotecnia, Economia Doméstica, Extensão Rural, Economia Aplicada, Entomologia, Solos, Química e Ecologia. Participaram também ao longo destes anos, profissionais de extensão rural, pesquisadores de entidades independentes e governamentais, representantes de órgãos municipais, estaduais e profissionais de diferentes áreas de atuação.

Quanto à organização dos oito ciclos, participaram 60 pessoas, sendo: pós-graduandos, professores e técnicos de diferentes formações (nutricionistas, educadores do campo, enfermeiro, economista doméstico e engenheiro agrônomo), além de graduandos. Até 2019 foram mediadores do Ciclo 109 pessoas, sendo 73 pósgraduandos, com formação em Nutrição, Administração, Jornalismo, Gestão de Cooperativas, Enfermagem, Farmácia, História e Ciências Biológicas (Figura 2).

Assim, a construção metodológica do "Ciclo de Discussão de Segurança Alimentar e Nutricional”, envolvendo diferentes atores, na formação e humanização do processo de aprendizagem pode ser entendida pela compreensão de Fernandes e colaboradores (2003) como sendo: 
O entendimento da necessidade de formar profissionais aptos a aprender e comprometidos com o enfrentamento dos graves problemas da nossa sociedade. A fundamentação teórica desse entendimento insere-se nas premissas da educação contemporânea: aprender a conhecer, aprender a fazer, aprender a viver juntos e aprender a ser.

\section{Envolvidos na organização}

- Pós-graduação $\square$ Graduação $\square$ Professores $₫$ Técnicos

66

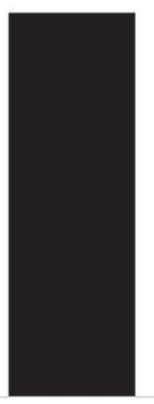

Envolvidos na mediação das discussões

- Pós-graduandos $\quad$ Professores $\square$ Técnicos

56

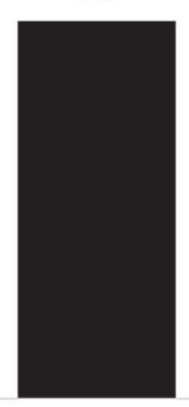

40

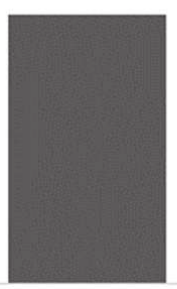

4

Figura 2. Perfil do público envolvido na organização e mediação do "Ciclo de Discussão de Segurança Alimentar e Nutricional" (2012-2019).

Fonte: Elaborado pelas autoras

Este processo de organização, considerando a abordagem pedagógica de ensino-aprendizagem, permite aos envolvidos se contextualizarem como agentes sociais que articulam as discussões inserindo-se em concepções críticas de questões envolvendo o contexto social da temática de SAN (MITRE et al., 2008).

A estruturação da metodologia do Ciclo insere-se no contexto das metodologias ativas que devem ser trabalhadas em processos de ensino-pesquisa-extensão. Assim, valoriza-se a educação formal e não formal dadas por desafios vivenciados por aqueles que trabalham a temática apresentada, destacando-se grupos e indivíduos dentro do contexto da autonomia relatada por Paulo Freire (FREIRE, 1982). Este intercâmbio faz com que mais pessoas tenham acesso às discussões construídas em sala de aula, podendo estas serem trabalhadas em contextos sociais distintos, em especial na valorização da prática profissional (SILVA, OLIVEIRA, 2019).

A formação e ocupação dos organizadores e mediadores dos Ciclos é heterogênea, permitindo o reconhecimento das diferentes características e demandas do público envolvido. Assim, a discussão ocorre de forma multidisciplinar, interprofissional e intersetorial, permitindo a compreensão das múltiplas vertentes relacionadas a SAN.

Esta dinâmica de construção de espaços de discussão e popularização dos saberes tem sido exitosa, em especial utilizando as dependências de instituições de ensino. Como exemplo têm-se os encontros realizados pelo "Café com Agroecologia" que é realizado nas dependências da UFV, Campus Viçosa. Neste espaço é realizada a divulgação da agroecologia buscando assim como o Ciclo, a heterogeneidade 
de formação profissional para contribuir com os diálogos críticos-reflexivos, como descrito por PratesJúnior e colaboradores (2017).

\section{Temas discutidos e reflexões na perspectiva das diretrizes da Política Nacional de Segurança Alimentar e Nutricional}

A partir da análise de similitude dos temas discutidos foi possível identificar a ocorrência e interligação entre as palavras, auxiliando na identificação da estrutura do conteúdo do corpo textual, ao longo das oito edições do "Ciclo de Discussão de Segurança Alimentar e Nutricional". Observa-se que, no centro da Figura 3 encontra-se o termo "Segurança Alimentar e Nutricional" e há três palavras que mais se destacam, em função da repetição na ocorrência das mesmas, sendo elas: "Alimento", "Saúde" e "Perspectiva". O termo do centro da figura se interliga de forma mais forte aos termos "Novo", "Agricultura Familiar", "Sistema Agroalimentar", "Campo" e "Agroecologia”. A espessura das arestas indica a força da conexão entre as palavras.

As discussões dos Ciclos têm abrangido diferentes temáticas no contexto da SAN, o que permite aos envolvidos no processo de organização e participação dos encontros, a familiarização e construção ativa do pensamento crítico em relação a variados temas (JACOBI, 2005). Espaços como os Ciclos de Discussão são ambientes que permitem que a comunidade interna e externa à universidade possa se familiarizar com as políticas e ações de promoção da SAN.

Algumas colocações demonstram que o ramo apresentado na Figura 3, dado pela "Insegurança Alimentar" conecta-se a palavra "agrotóxico" e contrapõe-se aos ramos dos termos: "sistema agroalimentar", "agroecologia”, “alternativa”, "incentivar”, "alimentação escolar”, dentre outros. A relação inversa da SAN com o agrotóxico ressalta os impactos negativos do mesmo na saúde humana e ambiental e é amplamente discutida na literatura e na atuação em campo (LOPES, ALBUQUERQUE, 2018). Já a relação congruente entre SAN, agricultura familiar, sistema alimentar e agroecologia, reforçam o incentivo a modelos de produção mais sustentáveis. Ressaltase também a importância de alimentos oferecidos no ambiente escolar, oriundos deste modelo de produção, que contribuem para a melhoria da oferta de uma alimentação saudável e adequada, promovendo a SAN (MARTINELLI, CAVALLI, 2019).

Considerando as relações observadas na Figura 3, ressalta-se que a alimentação saudável é uma ferramenta na prevenção de doenças e neste contexto a utilização de agrotóxicos viola este pressuposto, além de contribuir com a situação de Insegurança Alimentar e Nutricional, ferindo o Direito Humano à Alimentação Adequada e Saudável (BURITY et al, 2010). Além disso, a alimentação é uma necessidade básica influenciada por fatores sociais, nutricionais, econômicos e culturais (CARVALHO, ROCHA, 2011). Logo, a discussão desta temática perpassa por todas as áreas do conhecimento, de forma transdisciplinar, demonstrando a importância dos debates gerados nos Ciclos. 


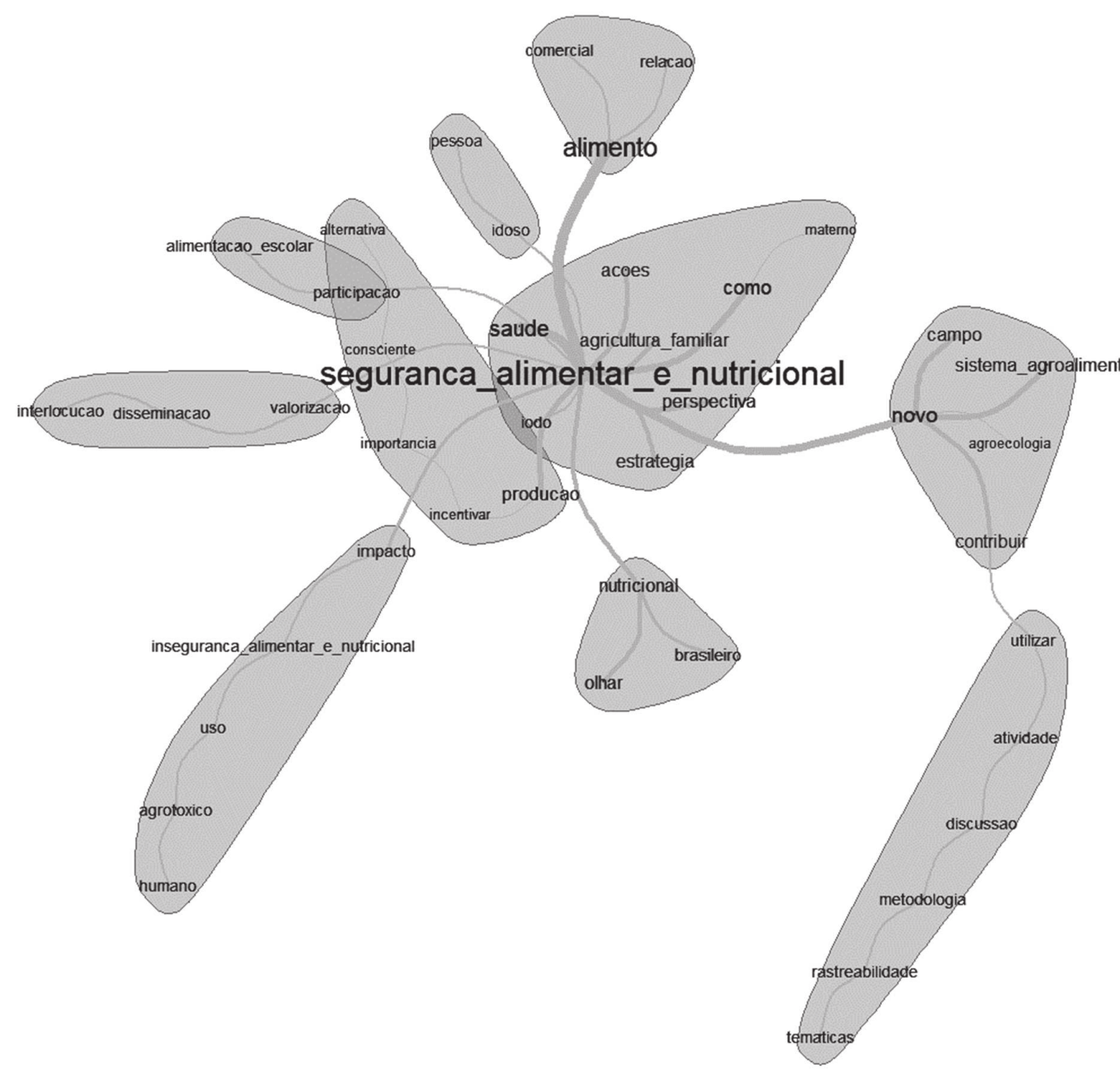

Figura 3. Análise de Similitude dos temas discutidos durante os "Ciclos de Discussão de Segurança Alimentar e Nutricional (2012-2019).

Fonte: Elaborado pelas autoras.

Todas as temáticas discutidas nos encontros dos Ciclos abarcam pelo menos um dos eixos das diretrizes da PNSAN (Figura 4). Os eixos mais debatidos foram o II " promoção do abastecimento e estruturação de sistemas sustentáveis e descentralizados, de base agroecológica, de produção, extração, processamento e distribuição de alimentos" e o V "fortalecimento das ações de alimentação e nutrição em todos os níveis da atenção à saúde, de modo articulado às demais ações de Segurança Alimentar e Nutricional”, seguidos dos eixos I "promoção do acesso universal à alimentação adequada e saudável, com prioridade para as famílias e pessoas em situação de Insegurança Alimentar e Nutricional” e VIII “monitoramento da realização do DHAA” (Figura 4).

Os temas apresentados durante os Ciclos incluem discussão e atualização sobre questões como histórico de SAN no Brasil e no mundo; conceito e determinantes desta situação; diferentes modelos de produção de alimentos e sua relação com a sustentabilidade ambiental; situaçãode SAN em grupos específicos, principalmente nos mais vulneráveis; programas governamentais conexos a SAN; teor nutricional e comercial dos alimentos e sua relação com a SAN; economia solidária; além de novas metodologias e alternativas de trabalho em campo relacionadas a SAN. 
Esta ampla discussão de temáticas que contemplam os eixos da PNSAN comprova que os debates ocorridos ao longo das edições dos Ciclos são temas relevantes, com apresentação de informações baseadas em evidências teóricas e em vivências dos apresentadores e mediadores.

Assim, a interação entre as diferentes áreas do conhecimento permite que o propósito dos Ciclos, em promover a troca de saberes entre sala de aula e a comunidade, seja alcançado. Destaca-se, que a questão da SAN é um tema amplamente debatido por órgãos nacionais e internacionais, a exemplo, a Organização das Nações Unidas para Alimentação e Agricultura (FAO), que em seus relatórios abordam a Insegurança Alimentar no mundo e seus determinantes (FAO, 2015; 2018).

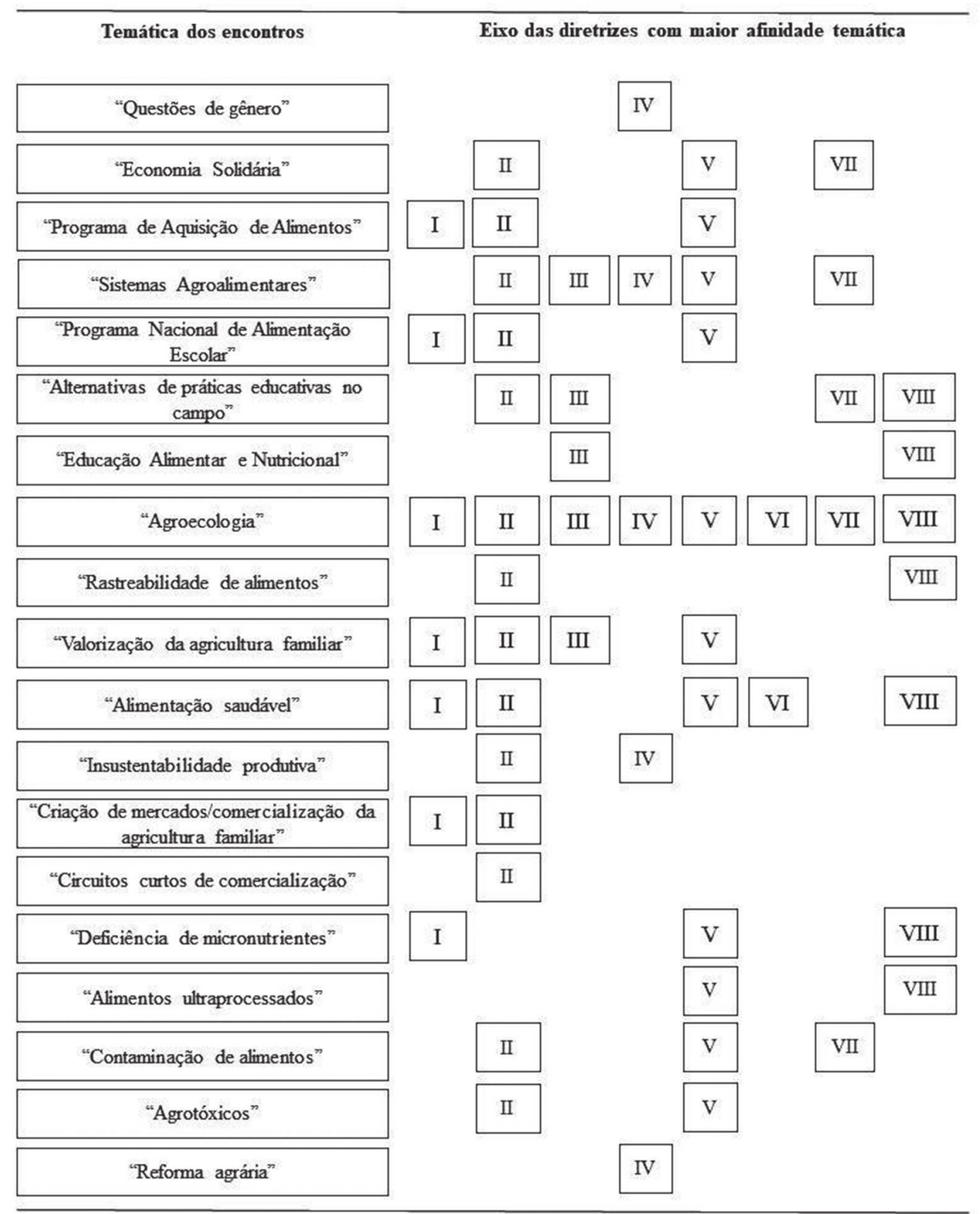

Figura 4. Categorização das temáticas dos Ciclos segundo os eixos das diretrizes da Política Nacional de Segurança Alimentar e Nutricional (PNSAN) (2012-2019).

Fonte: Elaborado pelas autoras 
Logo, os ambientes externos à sala de aula são considerados espaços de socialização e formação política, onde os indivíduos são impulsionados a serem críticos. Além disso, estes espaços contribuem para a sensibilização da realidade ao mesmo tempo em que visa buscar soluções para os diferentes problemas debatidos, como os apresentados na Figura 3 (MACEDO, MIRANDA, GARCIA, 2019).

Ressalta-se que para a resolução dos problemas faz-se necessário o ambiente para o diálogo, onde diferentes pontos de vista são ouvidos, seja em uma conversa individual ou coletiva. Estes locais proporcionam a troca de conhecimento e as Instituições de Ensino Superior constituem-se espaços de transformação social, construção de sujeitos ativos, críticos e profissionalmente qualificados (GOLDMEYER, 2019; SPONCHIADO, PAIM, 2019).

Enfatizamos que as discussões geradas na disciplina acerca de temas do cotidiano, que impactam na SAN permitem a formação de um cidadão crítico, tendo o professor um papel fundamental no desenvolvimento indagador do estudante, uma vez que o processo de aprendizagem exige comunicação e troca de conhecimento entre quem ensina e quem aprende (FREIRE, 1996).

Ações como as desenvolvidas nos encontros do "Ciclo de Discussão de Segurança Alimentar e Nutricional" reforçam a importância da tríade ensino, pesquisa e extensão, que são pilares do ensino em Universidades Públicas (COSTA, SANTOS, PRIORE, 2019). Este tipo de diálogo e troca de saberes demonstram o papel da universidade, representada pela integração de docentes, discentes, técnicos e profissionais de diferentes setores e áreas de atuação, como potencial transmissor do conhecimento para sociedade, tendo o Ciclo como uma importante ferramenta, já que este busca apresentar a prática como potencializadora de mudanças sociais (BARATA-SILVA, PEREIRA, GOMES, 2019).

\section{Desafios}

A realização dos Ciclos, de forma contínua, ao longo destes anos, tem como principal desafio manter a dedicação dos pós-graduandos que fazem a disciplina e do grupo de pós-graduandos que pesquisam sobre SAN, a disseminarem as discussões sobre a temática para a comunidade.

Outra questão, que também é um fator desafiador, mas também motivador para a realização dos encontros é a inclusão de atores externos a universidade que compartilham suas vivências e informações empíricas, trazendo assim a realidade para exemplificar a teoria apresentada nos vastos materiais científicos sobre SAN. Neste contexto, a participação de outros atores de SAN como agricultores familiares, cooperativistas, povos e comunidades tradicionais enriqueceriam o debate e deve ser incentivada, principalmente com maior divulgação dos encontros do Ciclo em espaços acessados pelos mesmos.

Destaca-se ainda, como fator desafiador, a necessidade de disseminar as informações discutidas nos Ciclos para além dos encontros presenciais. Assim, há grande motivação entre os integrantes da disciplina e envolvidos com a realização dos Ciclos para a elaboração de produção científica, em forma de resumos apresentados em congressos (LOPES et al., 2017), artigos publicados em revistas (MORAIS et al., 2014; 
MEDEIROS et al., 2020), capítulos de livro (DUTRA, 2019) e um livro elaborado a partir das discussões dos Ciclos (em construção), visando a expansão da discussão sobre SAN.

Em 2020, em função da pandemia do Coronavírus (COVID-19), enfrentamos o desafio de realizar o "IX Ciclo de Discussão de Segurança Alimentar e Nutricional" de forma on-line, utilizando plataformas de transmissão digitais. Oencontro virtual, realizado até o momento, seguiu a mesma metodologia dos anteriores, com a explanação de palestrantes e mediadores, e participação de um público heterogêneo de graduandos, pós-graduandos, docentes e profissionais que atuam na promoção da SAN. A novidade metodológica, utilizando meios diferentes do que estávamos acostumados, desafiou o grupo a desbravar esta tecnologia disponível e motivou a realizar novos eventos neste formato on-line, neste período adverso de pandemia. Mesmo diante de uma transmissão ao vivo o objetivo principal do Ciclo de gerar discussão, ampliando e integrando o conhecimento entre sala de aula e comunidade, foi atingido.

\section{CONCLUSÕES}

Ao longo destes oito anos, o “Ciclo de Discussão de Segurança Alimentar e Nutricional” tem socializado os temas estudados em sala de aula, promovendo a discussão da temática da SAN para além de uma disciplina, extrapolando essa discussão para a comunidade científica (graduandos, pós-graduandos, docentes e técnicos) e para quem atua na ponta (profissionais e técnicos de diferentes áreas de formação).

O estudo e divulgação dos pilares da SAN, contidos na PNSAN, bem como as atualizações e debates sobre esta temática são o destaque das discussões realizadas em todos os Ciclos.

Ações como estas são fundamentais para estimular o pensamento crítico para além da sala de aula e levantar questões que estimulem os debates em SAN, ampliando assim a promoção da discussão desta temática em diferentes grupos e espaços. Expandir as discussões geradas em disciplinas para eventos públicos, com a participação de estudantes e profissionais de diferentes áreas propicia a troca de saberes entre universidade e comunidade e deve, sempre que possível, ser incentivadas. Assim, reforça-se a missão da universidade pública com integração das ações de ensino, pesquisa e extensão.

\section{AGRADECIMENTOS}

Agradecemos a todas e todos que se envolveram no planejamento e realização dos encontros do "Ciclo de Discussão de Segurança Alimentar e Nutricional" ao longo destes anos, bem como aos nossos colegas que dedicam seu tempo à pesquisa e promoção da SAN, mesmo em tempos difíceis de manter viva e forte esta bandeira.

\section{REFERÊNCIAS}

AÇÃO BRASILEIRA PELA NUTRIÇÃO E DIREITOS HUMANOS (ABRANDH). O direito humano à alimentação adequada e o sistema nacional de segurança alimentar e nutricional. Brasília: ABRANDH, 2013. 263p. 
BARATA-SILVA, André Wagner; PEREIRA, Viviane Santos; GOMES, Luiz Antônio Augusto. Construindo saberes sobre desenvolvimento rural sustentável e agroecologia: uma experiência com extensão universitária uma experiência com extensão universitária. Revista Elo-Diálogos em Extensão, Viçosa, v. 8, n. 1, p. 1523, 2019.

BARDIN, Laurence. Análise de conteúdo. São Paulo-SP: Edições, 2011.

BRASIL. Lei no 11.346, de 15 de setembro de 2006. Cria o Sistema Nacional de Segurança Alimentar e Nutricional - SISAN com vistas em assegurar o direito humano à alimentação adequada e dá outras providências. Diário Oficial [da] República Federativa do Brasil, Brasília, DF, 2006.

BRASIL. Decreto ${ }^{\circ} 7.272$, de 25 de agosto de 2010. Regulamenta a Lei ${ }^{\circ} 11.346$, de 15 de setembro de 2006, que cria o Sistema Nacional de Segurança Alimentar e Nutricional-SISAN com vistas a assegurar o Direito Humano à Alimentação Adequada, institui a Política Nacional de Segurança Alimentar e NutricionalPNSAN, estabelece os parâmetros para a elaboração do Plano Nacional de Segurança Alimentar e Nutricional, e dá outras providências. Diário Oficial [da] República Federativa do Brasil, Brasília, DF, ago. 2010 .

BURITY, Valéria et al. Direito humano à alimentação adequada no contexto da segurança alimentare nutricional. Brasília: ABRANDH, 2010.

CARVALHO, Edilaine Oliveira; ROCHA, Emersom Ferreira. Consumo alimentar de população adulta residente em área rural da cidade de Ibatiba (ES, Brasil). Ciência e Saúde Coletiva, Rio de Janeiro, v.16, n.1, p.179-185, 2011.

COSTA, Bianca Aparecida Lima; SANTOS, Carla Cristina Balbino; PRIORE, Silvia Eloiza. Aproximando produção e consumo: a experiência do projeto de extensão "Quintal Solidário". Revista ELO - Diálogos em Extensão, Viçosa, v. 8, n. 1, p. 9-14, 2019.

DUTRA, Luiza Veloso. Diferentes metodologias para avaliação de segurança alimentar e nutricional em domicílios da área rural. In: CARMO, D. L. et al. Pesquisa em Agroecologia: conquistas e perspectivas. Viçosa-MG: Funarbe, 2019. Cap. 18.

FAO - ORGANIZAÇÃO DAS NAÇÕES UNIDAS PARAALIMENTAÇÃO E AGRICULTURA. El estado de la seguridad alimentaria y la nutrición en el mundo: Fomentando la resiliencia climática en aras de la seguridad alimentaria y la nutrición. Roma, 2018. 218p.

FAO - ORGANIZAÇÃO DAS NAÇÕES UNIDAS PARAALIMENTAÇÃO E AGRICULTURA. $E l$ estado de la inse guridad alimentaria en el mundo 2015. Cumplimiento de los objetivos internacionales para 2015 en relación con el hambre: balance de los desiguales progresos. Roma, 2015. 66p.

FERNANDES, Josicelia Dumêt et al. Estratégias para a implantação de uma nova proposta pedagógica na escola de enfermagem da Universidade Federal da Bahia. Revista Brasileira de Enfermagem, Brasília, v. 56, n. 4, p. 392-395, 2003.

FREIRE, Paulo. Considerações em torno do ato crítico de estudar. In: FREIRE, P. Ação Cultural para a Liberdade e outros escritos. 6. ed. Rio de Janeiro: Paz e Terra, 1982. p. 9-12. 
FREIRE, Paulo. Pedagogia da Autonomia: Saberes Necessários à Prática Educativa. 25ª ed. São Paulo: Paz e Terra, 1996.

GOLDMEYER, Marguit Carmem. Gestão educacional: no diálogo, o tecer coletivo dos fios de saberes. In: Anais do XXI Fórum de Estudos: leituras de Paulo Freire. KAODOINSKI, Fabiana et al. Caxias do Sul-RS: Educs, 2019.

JACOBI, Pedro Roberto. Educação ambiental: o desafio da construção de um pensamento crítico, complexo e reflexivo. Educação e Pesquisa, São Paulo, v. 31, n. 2, p. 233-250, 2005.

LOPES, Carla Vanessa Alves; ALBUQUERQUE, Guilherme Souza Cavalcanti. Agrotóxicos e seus impactos na saúde humana e ambiental: uma revisão sistemática. Saúde em Debate, Rio de Janeiro, v. 42, n. 117, p. 518-534, 2018.

LOPES, Sílvia Oliveira et al. Ciclos de discussão sobre segurança alimentar e nutricional: promovendo a troca de saberes entre sala de aula-comunidade. In: Anais do III Encontro Nacional de Pesquisa em Soberania e Segurança Alimentar e Nutricional. MEDEIROS, Maria Angélica Tavares et al. Curitiba-PR: Rede PENSSAN, 2017.

MACEDO, Sabrina Meirelle; MIRANDA, Pâmela Saraiva; GARCIA, Narjara Mendes. Concepções freireanas como proposição para a educação dos sentidos. In: Anais do XXI Fórum de Estudos: leituras de Paulo Freire. KAODOINSKI, Fabiana et al. Caxias do Sul-RS: Educs, 2019.

MARCHAND, Pascal; RATINAUD, Pierre. L'analyse de similitude appliquée aux corpus textuels: les primaires socialistes pour l'élection présidentielle française (septembre-octobre 2011). Actes des 11eme Journées internationales d'Analyse statistique des Données Textuelles. JADT, 2012, p. 687-699.

MARTINELLI, Suellen Secchi; CAVALLI, Suzi Barletto. Alimentação saudável e sustentável: uma revisão narrativa sobre desafios e perspectivas. Ciência e Saúde Coletiva, Rio de Janeiro, v. 24, n. 11, p. 42514262, 2019.

MEDEIROS, Natália Sant'Anna et al. Diverse food in urban gardens in the promotion of food and nutrition security in Brazil: a review. Journal of the Science of Food and Agriculture, v. 100, p. 1383-1391, 2020.

MINAYO, Maria Cecília de Souza. O desafio do conhecimento: pesquisa qualitativa em saúde. $13^{\circ}$ ed. São Paulo: HUCITEC, 2013.

MITRE, Sandra Minardi et al. Metodologias ativas de ensino-aprendizagem na formação profissional em saúde: debates atuais. Ciência e Saúde Coletiva, Rio de Janeiro, v. 13, n. 2, p. 2133-2144, 2008.

MORAIS, Dayane de Castro et al. Insegurança alimentar e indicadores antropométricos, dietéticos e sociais em estudos brasileiros: uma revisão sistemática. Ciência e Saúde Coletiva, Rio de Janeiro, v. 19, n. 4, p.1475-1487, 2014.

MORAIS, Dayane de Castro; SPERANDIO, Naiara. In: NAVES, Bruno Torquato de Oliveira; FERNANDES, Fabíola Ramos; NASCIMENTO, Simone Murta Cardoso. Repercussões éticas e jurídicas da genética: Apontamentos sobre os impactos na saúde e no meio ambiente. Coleção Diálogos sobre meio ambiente. V.III. ARRAES Editores. 2016. p-66-79. 
NASCIMENTO, Amália Leonel; ANDRADE, Sonia Lúcia L. Sousa. Segurança alimentar e nutricional: pressupostos para uma nova cidadania? Ciência e Cultura, Campinas, v. 62, n. 4, p. 34-38, 2010.

PRATES-JUNIOR, Paulo et al. Café com Agroecologia: integrando conhecimentos. Revista ELO - Diálogos em Extensão, Viçosa, v. 6, n. 3, p. 1-11, 2017.

PRIORE, Silvia Eloiza et al. Nutrição Social. Série Didática. Editora UFV. 156p.

RATINAUD, Pierre. IRAMUTEQ: Interface de R pour lês analyses multidimensionnelles de textes et de questionnaires [Computer Software]. 2009.

SILVA, Emerson Alcides; OLIVEIRA, Edgard Leite. Extensão universitária e formação discente: características do processo educativo da incubadora tecnológica de cooperativas populares: características do processo educativo da Incubadora Tecnológica de Cooperativas Populares. Revista Elo - Diálogos em Extensão, Viçosa, v. 8, n. 1, p. 33-48, 2019.

SPONCHIADO, Laercio Francisco; PAIM, Marilane Maria Wolff. A escola como um espaço possível na construção da emancipação do sujeito. In: Anais do XXI Fórum de Estudos: leituras de Paulo Freire. KAODOINSKI, F. et al. Caxias do Sul-RS: Educs, 2019.

Data de Submissão: 22/06/2020 Data de Aceite: 14/08/2020 